\title{
Team Activity of Robot Competition of Simulated Robot in World Robot Summit 2020
}

\author{
Daichi Shima ${ }^{1}$, Tomoyuki Furukawa ${ }^{1}$, Ryuma Aoba ${ }^{1}$, Ayato Ohashi ${ }^{1}$, Kota Tsuruno $^{1}$, and Keitaro Naruse ${ }^{1 *}$ \\ ${ }^{1}$ The University of Aizu, Department of Computer Science and Engineering, Aizu-Wakamatsu, Japan
}

\begin{abstract}
World Robot Summit (WRS) has several robot competitions, and we will participate it in the infrastructure and disaster response category. Participating teams develop their robot system by teleoperation and/or autonomous operation and run it in a set of courses modelling and simplifying disaster responding situations. The authors will attend the challenge of the tunnel disaster response and recovery, in which we are requested to achieve an investigation and rescue scenario of a tunnel fire with simulated robots. As preparation, we develop simulated robot models and corresponding software as a team. In this article, we report out activity to the robot competition and student's project-based learning by joining it.
\end{abstract}

\section{Introduction}

Generally. Robot simulation is used to check what kind of problems will occur in advance with a computer before an actual robotic system is operated in a real workspace. In addition. It is also used for training of new robot operators, as well as designing to test robot structure and motion before we build it physically.

In our laboratory, we have developed the actual disaster response robot as shown in Fig. 1 and Fig. 2, as well as corresponding simulated robot as in Fig. 3 and Fig. 4. We use the simulated robot mainly for developing robot software before implementing to the actual robot.

On the other hand, for open innovation of robot technologies, robot competitions have been held worldwide. We are registered and will join to the infrastructure, and disaster response category in World Robot Summit 2020 (WRS2020) [1], which is postponed in the next year due to COVID-19.

In this paper, we report on our activity for the competition by the simulated robots as an instance of project-based learning in robotics.

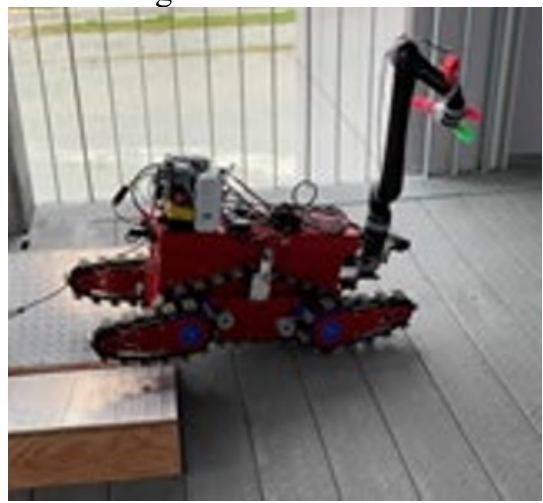

Fig. 1. Small disaster response robot named Spider 2020

\footnotetext{
* Corresponding author: naruse@u-aizu.ac.jp
}

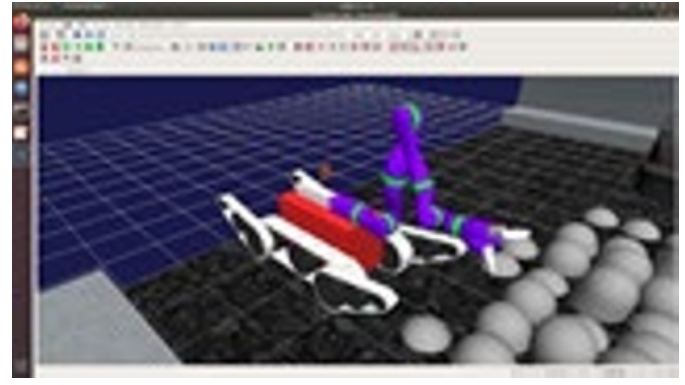

Fig. 2. Simulated robot model of Spider 2020.

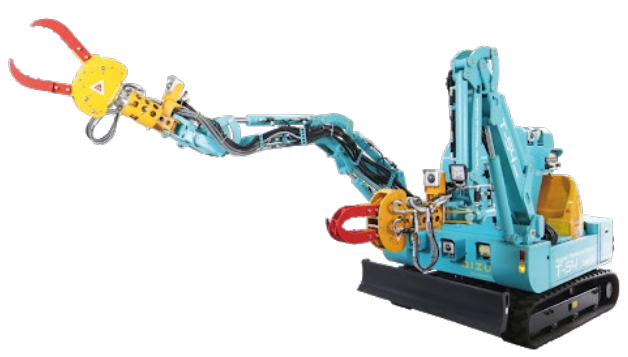

Fig. 3. Large disaster response robot named Enryu.

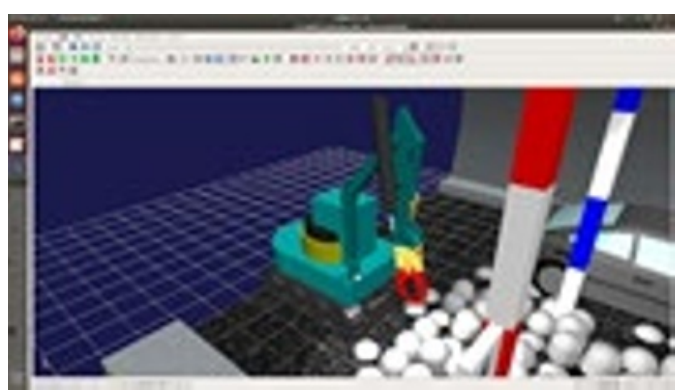

Fig. 4. Simulated robot model of Enryu. 


\section{Development}

For attending the robot competition by simulated robots, we have been working on both model and software development as a student team. Here we present our tools and process of development.

\subsection{Development environment}

Table 1 shows our development environment of simulated robots. We use the robot simulator called choreonoid [2], which is designated by the regulation of the competition, under a recommended OS of Ubuntu 18.04 LTS. The simulator introduces a numerical calculation engine named AGX Dynamics [3] for robot motion dynamics.

We develop our software mainly by $\mathrm{C}++$ language with linear algebra SDK called Eigen [4], and some tools are implemented in Python. As communicating with other services, we use the robot middleware called ROS [5]. We introduce github [6] for code sharing and Google drive for other document sharing.

We employ a gamepad named dual shock 4 for operating the simulated robots.

Table 1. Development environment of simulated robots.

\begin{tabular}{|l|l|}
\hline OS & Ubuntu 18.04 LTS \\
\hline $\begin{array}{l}\text { Programming } \\
\text { Languages }\end{array}$ & C ++ , Python \\
\hline Simulator & Choreonoid \\
\hline Middleware & ROS (Robot Operating System) \\
\hline Dynamics engine & AGX Dynamics \\
\hline SDK & Eigen \\
\hline Code share & Github, Google drive \\
\hline Other devices & Dual Shock 4 \\
\hline
\end{tabular}

\subsection{Development process}

We mainly operate Choreonoid through ROS interface. First, we develop a robot model using CAD or other tools. Then we develop the software to control the model according to choreonoid SDK and check the simulation feasibility on it. After that, we check the coordination with external visualizer with ROS interface and complete the development.

\section{Task and Problem}

In this section, we describe instances of courses and required functionalities of the simulated robots, showing those in the stage gate qualification of WRS2020.

\subsection{Task}

For tasks, there are four tasks that respond to tunnel disasters.

\section{(1) Investigation and rescue of vehicles and surroundings}

Tasks:
Investigation around, inside, and outside of the vehicle; breaking down doors; investigation and rescue of people in need of help (Fig. 5).

Points:

Can the robot perform a visual inspection in a small, intricate, and dark place? How carefully can it handle the human body?

\section{(2) Securing the route}

Tasks:

Moving obstacles out of the path, trans-shipment, stick pulling, preparing for blasting (Fig. 6).

Points:

How to handle an L-shaped obstacle with an unknown canter of gravity.

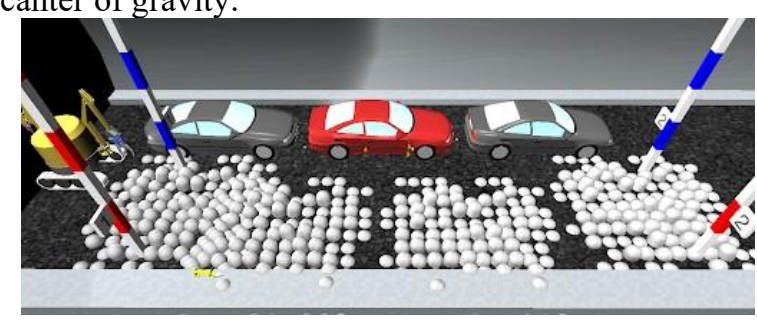

Fig. 5. Task (1) Investigation and rescue of vehicles and surroundings.

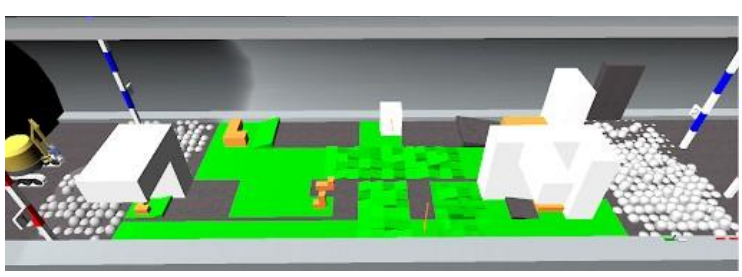

Fig. 6. Task (2) Securing the route.

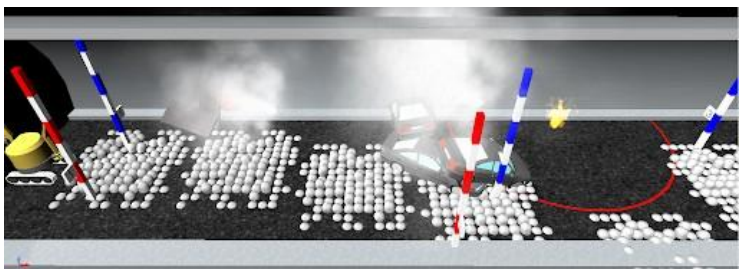

Fig. 7. Task (3) Firefighting work.

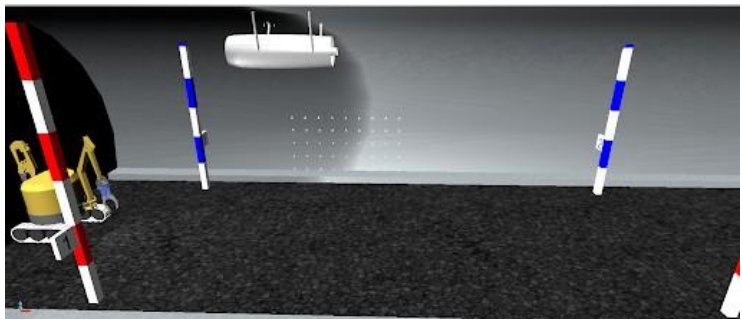

Fig. 8. Task (4) Inspection of the tunnel wall and ancillary equipment.

\section{(3) Firefighting work}

Tasks:

Hose withdrawal, hose and nozzle connection, valve operation, digestion (Fig. 7).

Points:

Ability to handle a variety of digestion tasks (e.g., handling of hoses as flexible objects, connection of 
nozzles and hoses, etc.), ability to deal with the reaction force of water discharge.

\section{(4) Inspection of the tunnel wall and ancillary equipment}

Tasks:

Checking jet fan bolts, tunnel wall and road surface Point:

Is there a large space capable of handling the inspection of the walls of the tunnel itself and ancillary equipment such as jet fans? (Fig. 8)

\subsection{Problems}

Most of the tasks can be solved by our first robot models. However, the following two issues are required to develop a new robot model. We have solved it by developing a new camera device called the end-scope camera.

\section{First issue:}

In the task 1 and task 4 , there are many cylindrical objects, and at the end of them, there are QR codes which are required to be viewed and decoded by the camera mounted on the robot. They needed to be seen and read by a camera mounted on the robot, a task that was nearly impossible with the existing robot model.

\section{Second issue}

In task 4, it is difficult to extend the arm up to the QR code present in the top of the jet fan due to the robot size. On the other hand, we can introduce a drone to measure it, but it is also difficult to read the QR code from it due to position stability.

\subsection{Solution}

To solve the above problems, we introduced an idea of an end-scope camera as seeing 365-degree view, which is shown in Fig. 9. It has four joints, which are controlled independently. In addition, we have developed a robot arm controller with inverse kinematics, which allows us to move a hand tip to designated position automatically.

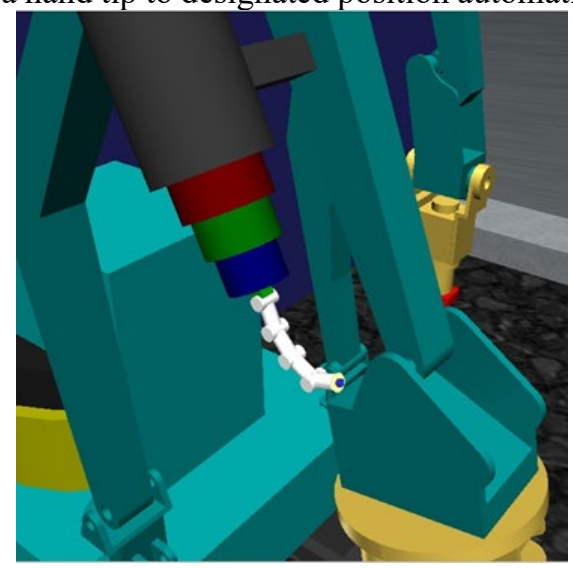

Fig. 9. End-scope camera (White device).
As a result, we are able to solve most of the problems in task 4. However, it is still difficult to adjust the camera to find and focus to a QR code only looking at a camera image. We are currently developing a robot arm controller to solve it.

\section{Activity}

WRS2020 was scheduled to be held in July 2020, but it was postponed due to COVID-19, and it is now scheduled to be held in May in the next year. Therefore, we have organized a demonstration event by us in October in this year, for demonstrating our robot model and controller, and handing them over to team members in the next year.

We have made two teams: the first team is made by fourth-year students and the other one is by third-year students in our university.

The demonstration event is composed of two phases: The first one is offline challenge. We have organized a review committee separated from participating teams. The committee has created a set of courses, opened it in github, asked the participating teams to challenge them by their PCs and to submit the result.

The other one is online symposium by the teams to share their experience, which is done with zoom.

\section{1 Offline challenge}

In the challenge, it is required to perform tele-operation, which means a robot operator can only observe a workspace from a set of cameras attached to a robot and cannot see a perspective view of a workspace.

We have designed two courses as follows with less difficult than the WRS courses because we have less experienced team in this event.

(1) Stage-Gate 1 for large robot

Tasks: Operating a large-size simulated robot to go through a narrow room without colliding with obstacles

(Fig. 10).

(2) Stage-Gate 2

Tasks: Operating a large-size simulated robot to pushing a L-shaped obstacle to be aligned at a designated area (Fig. 11).

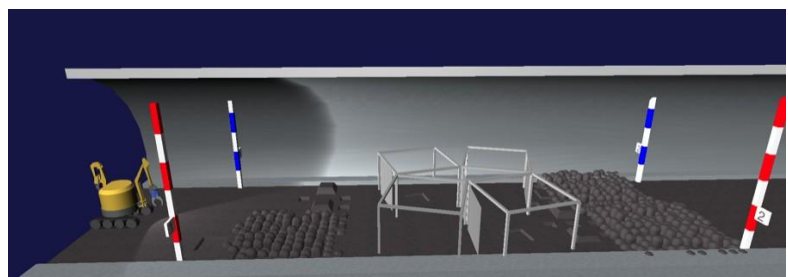

Fig. 10. SG1L course.

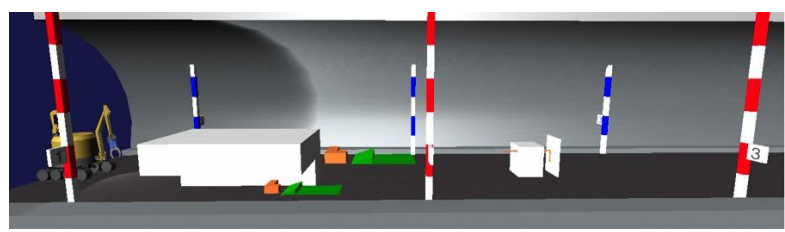

Fig. 11. SG2 course. 


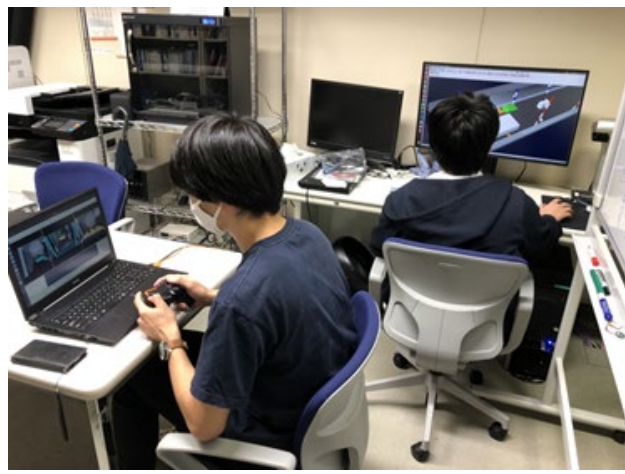

Fig. 12. Snapshot of task challenging.

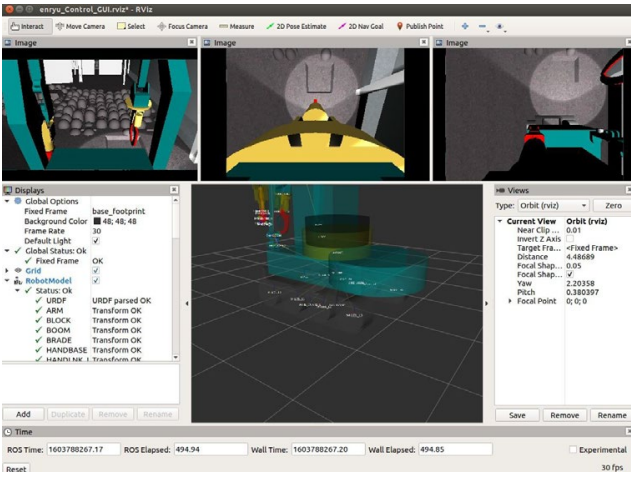

Fig. 13. Operator's screen.

To ensure that there are no irregularities in the task attack, the operation PC and the monitoring PC are separated, with one person acting as operator and one as supervisor. On the operation PC, a user interface utilizing the ROS feature Rvis is used to control the entire operation. The monitoring PC observes the entire field using choreonoid and monitors it for judging if the task is performed well. A snapshot of task challenging, and operator's screen is shown in Fig. 12 and Fig. 13, respectively.

\section{2 Online-symposium}

We have two teams joined in the offline challenge. On the other hand, for open innovation of robot technology, we should have an opportunity to share experience of teams joined in the offline challenge. Therefore, we have arranged an online symposium by team members, competition officials, who has designed the offline challenge course, and several experts on robot simulator for giving valuable comments to the team members.

In the symposium, we have two student teams, and they have presented their strategy to attack the challenge, have showed their video of challenging, and have given comments from attendees.

On the other hand, a whole process of students' activity can be regarded as a project-based learning. Process (PBL). We will summarize comments from the students as follows.

\subsubsection{Learning effect of the team of 4th grade students}

The fourth-year undergraduate team had formed for WRS2020, which was scheduled for this year, and was focused on the operation and development of the system. We were able to clear most of the tasks. As for future activities, we proposed that we will focus on handing over the technology to junior members.

\subsubsection{Learning effect of the team of 3rd grade students}

The team of third-year undergraduates spent about a month preparing for the demonstration, focusing on the activities of the operators and participating as a new team in preparation for the WRS, which is scheduled to take place next year. They presented the improvements of the current system and user interface as an operator in the presentation of their results and presented their goals and improvements for the WRS.

\subsubsection{Learning activities among students}

At the online symposium, we consider the handing over of technology to the younger students to be the most important thing, so we hold a study session focusing on choreonoid and ROS among the students. Specifically, they are taught how to create models and development procedures.

\section{Conclusion}

In this paper, we have reported our activity of development and team training as an instance of projectbased learning in robotics.

\section{References}

1. Tunnel Disaster Response and Recovery Challenge in World Robot Summit 2020, https://worldrobotsummit.org/en/wrs2020/challeng e/disaster/tunnel.html

2. Choreonoid, https://choreonoid.org/ja/

3. AGX Dynamics, https://www.algoryx.se/agxdynamics/

4. Eigen, http://eigen.tuxfamily.org/

5. ROS, https://www.ros.org/

6. Github, https://github.com/ 